Carmen García-Ruiz ${ }^{\mathrm{a}}$, Román Andrés ${ }^{\text {b), }}$ Jose Luis Valerab), Fernando Laborda ${ }^{\text {b), }}$ María Luisa Marina ${ }^{\mathrm{a}, \mathrm{b}}$ )

a) Departamento de Química Analítica, Facultad de Química, Universidad de Alcalá, Ctra. Madrid-Barcelona Km. 33.600, 28871 Alcalá de Henares (Madrid), Spain

b) Centro de Tecnología de los Alimentos y Servicios

Biosanitarios, Universidad de Alcalá, Ctra. Madrid-Barcelona Km. 33.600, 28871 Alcalá de Henares (Madrid), Spain

\section{Monitoring the stereoselectivity of biodegradation of chiral polychlorinated biphenyls using electrokinetic chromatography}

\begin{abstract}
A study of the biodegradation of several chiral polychlorinated biphenyls (PCBs, IUPAC numbers $45,88,91,95,136,144,149$, and 176) by a naturally occurring soil bacterium as well as the stereoselectivity of such process has been performed using Electrokinetic Chromatography with cyclodextrins (CD-EKC). In order to achieve the analysis of chiral PCBs, a CD-EKC system based on $50 \mathrm{mM}$ 2-morpholinoethanesulfonic acid (MES) buffer ( $\mathrm{pH}$ 6.5) containing $2 \mathrm{M}$ urea, $20 \mathrm{mM}$ carboxymethylated $\gamma$ cyclodextrin (CM- $\gamma-C D$ ), and $10 \mathrm{mM} \beta$-cyclodextrin $(\beta-C D)$ was used. First, the percentage degradation of $\mathrm{PCBs}$ by the microorganism used in this work was measured for different incubation times (22, 46, 71, 93, 165, 213, and 262 hours). The results showed a high degree of biodegradation (from $61 \%$ to $94 \%$ ) for the eight PCBs studied after 262 hours of incubation with the microorganism, degradation being favoured for the least chlorinated congeners. Secondly, measurement of the atropisomeric ratio of the PCB atropisomers during the biodegradation process revealed no significant variation of the atropisomeric ratios with the degradation time, indicating a nonstereoselective degradation of PCBs by the microorganism.
\end{abstract}

Key Words: Chiral polychlorinated biphenyls; Stereoselectivity; Biodegradation; Electrokinetic chromatography

Received: May 21, 2001; revised: October 23, 2001; accepted: October 25, 2001

\section{Introduction}

Polychlorinated biphenyls (PCBs) are aromatic compounds that constitute an important class of priority pollutants classified by the U.S. Environmental Protection Agency (EPA) as carcinogens [1, 2]. Biphenyls with bulky atoms in ortho positions of the phenyl rings can be defined as molecules with axial chirality, in which rotation about the axis is hindered by steric congestion. In addition, if such a rotational energy barrier is high enough to resist racemisation at room temperature, the atropisomers will be conformationally stable [3]. Among the 78 PCBs that possess axial chirality, only 19 are stable towards racemisation at room temperature.

The enantiomers of chiral compounds may have different biological and toxicological effects. Therefore, enantioselective discrimination of chiral pollutants in environmental samples may yield information about possible degrada-

Correspondence: Prof. María Luisa Marina, Departamento de Química Analítica, Facultad de Química, Universidad de Alcalá, Ctra. Madrid-Barcelona Km. 33.600, 28871 Alcalá de Henares (Madrid), Spain.

E-mail: mluisa.marina@uah.es

Fax: +34 918854971

Abbreviations: Polychlorinated Biphenyls, PCBs; Electrokinetic Chromatography, EKC. tion pathways $[4,5]$. The main routes for the environmental degradation of PCBs are microbiological processes besides abiotic processes [6].

To date, the technique most widely employed to analyse achiral or chiral PCBs has been gas chromatography (GC) [7-13]. Nevertheless, capillary electrophoresis (CE) has experienced enormous growth in the field of chiral separations because of its flexibility and high separation efficiency [14]. Cyclodextrins (CDs) are known to exhibit strong chiral recognition towards several chiral PCBs present in commercial PCB formulations resulting from interactions such as the formation of inclusion complexes. Thus, cyclodextrin-modified micellar electrokinetic chromatography (CD-MEKC) has been employed to achieve the chiral separation of twelve chiral PCBs (IUPAC numbers $45,84,88,91,95,132,136,139,149,171,183$, and $196)$ using sodium dodecyl sulfate (SDS) micelles with $\gamma$ cyclodextrin ( $\gamma-C D)$ [15] or mixtures of $\beta$-cyclodextrin ( $\beta$ CD) and $\gamma$-CD [16]. The use of CD-MEKC with bile salts (sodium cholate) mixed with SDS micelles and $\gamma$-CD [17] enabled the chiral separation of twelve PCBs (IUPAC numbers 45, 84, 88, 91, 95, 131, 135, 136, 139, 144, 175, 176). Under the above-mentioned conditions it was possible to separate the atropisomers of eight or nine PCBs in mixtures in about $35 \mathrm{~min}$. Nevertheless, when bile salts alone [18] were used in CD-MEKC only the individual 
chiral separation of PCB 84, 95, and 176 was possible. Nevertheless, electrokinetic chromatography (EKC) using a dual cyclodextrin system has recently been employed for the rapid chiral separation of thirteen of the 19 PCBs stable at room temperature (PCBs 45, 88, 91, 95, 131, $132,136,139,144,149,171,176,197)$ [19]. Under these conditions, the chiral separation of the atropisomers of up to five chiral PCBs was possible in less than $12 \mathrm{~min}$. Therefore, this system seems to be the most appropriate in order to check the chirality of a major number of chiral PCBs using the shortest analysis time.

In this work, a naturally occurring soil bacterium isolated from a soil with a history of contamination has been employed to perform the biodegradation of eight chiral PCBs (IUPAC numbers 45, 88, 91, 95, 136, 144, 149, and 176) with different degrees of chlorination. In order to obtain information related to the stereoselectivity of the biodegradation of PCBs by such bacteria, a study on the atropisomeric ratio of the baseline resolved atropisomers has been performed during the biodegradation process and the results are reported herein.

\section{Experimental}

\subsection{Reagents}

All reagents employed were of analytical grade. 2-Morpholinoethanesulfonic acid (MES), dimethylformamide (DMF), and sodium hydroxide were supplied by Merck (Darmstadt, Germany); $\beta$-cyclodextrin $(\beta-C D)$ and urea were from Fluka (Buchs, Switzerland); carboxymethylated $\gamma$-cyclodextrin (CM- $\gamma-C D$, degree of substitution (DS) ca. 3) was obtained from Cyclolab (Budapest, Hungary). Water purified through a Milli-Q system from Millipore (Bedford, MA, USA) was employed to prepare the solutions. All solutions were filtered prior use through $0.45 \mu \mathrm{m}$ pore size disposable nylon filters from Scientific Resources (Eatontown, NJ, USA)

Acetone, dichloromethane, and hexane of pestipure grade from sds (Peypin, France) were used in glassware cleaning and extraction process

The eight PCBs studied were supplied by Dr. Ehrenstorfer Reference Materials (Augsburg, Germany). These compounds and their IUPAC numbers (between parentheses) according to the Ballschmiter nomenclature [20] are the following: 2,2',3,6-tetrachlorobiphenyl (PCB 45), 2,2',3,4,6-pentachlorobiphenyl (PCB 88), 2,2',3,4',6-pentachlorobiphenyl (PCB 91), 2,2',3,5',6-pentachlorobiphenyl (PCB 95), 2,2',3,3',6,6'-hexachlorobiphenyl (PCB 136), 2,2',3,4,5',6-hexachlorobiphenyl (PCB 144), 2,2',3,4', 5',6-hexachlorobiphenyl (PCB 149), and 2,2',3,3',4,6,6'-heptachlorobiphenyl (PCB 176).

\subsection{Apparatus}

A HP3D CE system (Hewlett-Packard, Waldbronn, Germany) equipped with an on-column diode array detector (DAD) and a HP 3D-CE Chemstation software was used. Separations were performed using uncoated fused-silica capillaries of inner diameter (ID) $50 \mu \mathrm{m}$ and outer diameter (OD) $375 \mu \mathrm{m}$, purchased from Composite Metal Services LTD (Worcester, England). The capillaries had a total length of $58.5 \mathrm{~cm}$ and $50 \mathrm{~cm}$ to the detector. The capillary temperature was set at $45^{\circ} \mathrm{C}$ and UV detection was performed at $230 \mathrm{~nm}$.

Electrolytic solutions were degassed in an ultrasonic bath KM from Raypa (Barcelona, Spain). A 654 pH-meter from Metrohm (Herisau, Switzerland) was employed to adjust the $\mathrm{pH}$ of the separation buffers.

A Büchi Rotavapor R-134 (Flawil, Switzerland) and a Pierce Reacti-Vap model 18780 evaporating unit (Rockford, Illinois, USA) were used to totally evaporate the hexane of the extracts.

\subsection{Procedure}

The strain (MS3-02) used in this work was isolated from samples of soil of the Comunidad de Madrid. First, the degradation activity of PCBs by this strain was checked by analysing a commercial mixture of PCBs (Arochlor 1242) by gas chromatography [21]. This strain has been characterised and identified by the Spanish Type Culture Collection as Jonibacter sp.

The culture conditions of the microorganism were as follows: $10 \mathrm{~mL}$ of modified phosphate-buffered mineral salts medium (PASM) with biphenyl were inoculated with the culture strain at $28^{\circ} \mathrm{C}$ during 48 hours. Then the culture was centrifuged (4000 rpm for $15 \mathrm{~min}$ ) and the precipitate was washed three times with a sodium chloride solution $(0.9 \% \mathrm{w} / \mathrm{v})$. Finally, a suspension of the microorganism in a solution of sodium chloride $(0.9 \% \mathrm{w} / \mathrm{V})$ was prepared by adding the microorganism until one unit of absorbance was measured at $630 \mathrm{~nm}$.

The samples were prepared using the incubation medium (10 mL of PASM) with $1 \mathrm{~mL}$ of a solution of hexane containing a mixture of two PCBs (149-95, 88-136, 45-91, 144-176) at a concentration of $0.5 \mathrm{ppm}$ for each PCB. Hexane was evaporated at room temperature prior to the addition of $0.5 \mathrm{~mL}$ of the above-mentioned microorganism suspension. In the samples the microorganism was inactivated by the addition of $70 \mu \mathrm{L}$ perchloric acid after the corresponding incubation times whereas for the control samples the microorganism was inactivated immediately.

After inactivation of the bacterium with perchloric acid the hydrophobic PCBs were extracted with hexane $(4 \times 10 \mathrm{~mL})$ and dried with anhydrous sodium sulfate. The 
solvent was removed in a rotary evaporator. The extract was redissolved with hexane $(4 \times 0.5 \mathrm{~mL})$ and placed in 2$\mathrm{mL}$ vials. Hexane was then evaporated to dryness under a nitrogen stream. The final extract containing PCBs was reconstituted with DMF $(50 \mu \mathrm{L})$ prior to $\mathrm{CE}$ analysis. Extractions were performed in duplicate.

The extent of recovery for the extraction was checked for PCB 91 as follows: $1 \mathrm{~mL}$ of PCB 91 in hexane (4.8 ppm) was added to $10 \mathrm{~mL}$ of PASM. After evaporation of hexane at ambient temperature the extraction was carried out as described above. The final extract was redissolved in $150 \mu \mathrm{L}$ of DMF containing 200 ppm of PCB 84 as internal standard. A calibration mixture was prepared evaporating to dryness $1 \mathrm{~mL}$ of a solution in hexane of PCB 91 (4.8 ppm) and adding $150 \mu \mathrm{L}$ of the above internal standard solution. The extent of recovery ranged from 77 to $75 \%$ when two individual experiments were injected in duplicate.

The separation buffer was prepared dissolving the appropriate amount of MES, CD derivatives, and urea in water to achieve a final concentration 50 mM MES-2 M urea$20 \mathrm{mM} \mathrm{CM}-\gamma-\mathrm{CD}-10 \mathrm{mM} \beta-\mathrm{CD}$ prior to the addition of water [19]. Finally, the $\mathrm{pH}$ was adjusted to 6.5 with $1 \mathrm{M}$ or $0.1 \mathrm{M}$ sodium hydroxide solution prior to making up the final volume.

Before first use, a new capillary was rinsed with $1 \mathrm{M}$ $\mathrm{NaOH}$ for $30 \mathrm{~min}$, followed by a 30-minute rinse with water. Between runs, the capillary was conditioned with DMF for $4 \mathrm{~min}, 0.1 \mathrm{M} \mathrm{NaOH}$ for $4 \mathrm{~min}$, and separation buffer for $4 \mathrm{~min}$. Injections were made by pressure (50 mbar for $1 \mathrm{~s}$ or $10 \mathrm{mbar}$ for $1 \mathrm{~s}$ for recovery determination). A separation voltage of $20 \mathrm{kV}$ was used and the detection took place at $230 \mathrm{~nm}$. This conditioning method with DMF, solvent used to dissolve the PCBs, was used to obtain good peak shapes and reproducible retention data. Two injections were performed for each sample.

\subsection{Calculations}

In this work, corrected areas have been used in order to compensate fluctuations in electrophoretic conditions and to obtain a good reproducibility of data. Corrected areas were obtained by dividing the raw area by the migration time [22].

\section{Results and discussion}

Electrokinetic chromatography using a dual cyclodextrin system based on a $50 \mathrm{mM}$ 2-morpholinoethanesulfonic acid (MES) buffer ( $\mathrm{pH}$ 6.5) containing $2 \mathrm{M}$ urea, $20 \mathrm{mM}$ carboxymethylated $\gamma$-cyclodextrin $(\mathrm{CM}-\gamma-\mathrm{CD})$, and $10 \mathrm{mM} \beta$-cyclodextrin ( $\beta$-CD) at $20 \mathrm{kV}$ and $45^{\circ} \mathrm{C}$, using an on-column diode array detector at $230 \mathrm{~nm}$ for detection [19], has been employed to monitor the biodegradation of eight chiral PCBs by a naturally occurring soil bacterium and to study the stereoselectivity of such process.

\subsection{Biodegradation of chiral PCBs}

A study on the biodegradation process by the naturally occurring bacterium has been performed for eight chiral PCBs (IUPAC numbers 45, 88, 91, 95, 136, 144, 149, and 176). These PCBs have been analysed in mixtures of two congeners (149-95; 88-136; 176-144; and 91-45) baseline separated by a chiral system which recognises the atropisomers of each congener. Table 1 shows the difference between the average migration times for the atropisomers of each congener in each mixture of two PCBs and the chiral resolution of each PCB for control samples. It can be observed that atropisomers of seven PCBs are baseline resolved $(R s>1.53)$ whereas atropisomers of PCB 176 are only partially separated (Rs = 0.47).

In order to evaluate the extent of the degradation of PCBs at different times of incubation the total peak area for each congener (addition of the area of the peak of the firstmigrating atropisomer and the area of the peak of the second-migrating atropisomer) was measured in the corresponding electropherogram at different incubation times.

Table 1. IUPAC numbers (according to Ballschmiter and Zell nomenclature [20]) and pairs of PCBs studied in this work together with the resolution for PCB atropisomers and differences of migration times for each pair of congeners considered in the control samples (0 hours of incubation).

\begin{tabular}{cccc}
\hline $\begin{array}{c}\text { PCBs IUPAC } \\
\text { number }\end{array}$ & $\begin{array}{c}\text { Chiral } \\
\text { resolution }\end{array}$ & $\begin{array}{c}\text { Pairs of } \\
\text { PCBs }^{\text {b) }}\end{array}$ & $t_{2}^{\prime}-t_{1}{ }^{\text {c })}$ \\
\hline 45 & 2.67 & $149-95$ & 2.09 \\
88 & 1.62 & & \\
91 & 2.32 & $88-136$ & 0.22 \\
95 & 4.16 & & \\
136 & 3.72 & $176-144$ & 0.76 \\
144 & 1.91 & & \\
149 & 1.53 & $91-45$ & 1.79 \\
176 & 0.47 & & \\
\hline
\end{tabular}

a) Mean value of four determinations calculated as $R s=1.18\left(t_{2}-t_{1}\right) /\left(w_{1}+w_{2}\right)$ where $t_{1}$ and $t_{2}$ are the migration times of the two atropisomers of a PCB and $w_{1}, w_{2}$ correspond to middle high width of the corresponding peaks.

b) Pairs of PCBs studied following the migration order.

c) $t_{2}^{\prime}-t_{1}^{\prime}=\left[\left(t_{3}+t_{4}\right) / 2\right]-\left[\left(t_{1}+t_{2}\right) / 2\right]$. $t_{1}$ and $t_{2}$ are the migration times in minutes for the atropisomers of the first-migrating PCB.

$t_{3}$ and $t_{4}$ are the migration times in minutes for the atropisomers of the second-migrating PCB. 


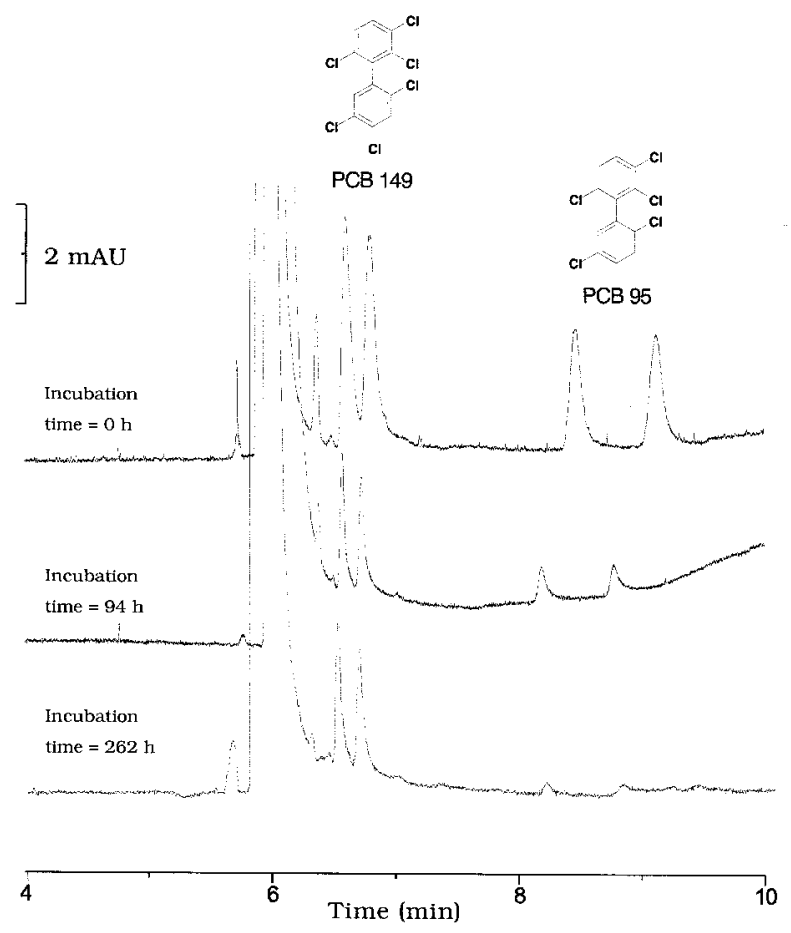

Figure 1. Electropherograms obtained during the biodegradation of a mixture of PCBs 149 and 95 at three different incubation times $(0,94$, and 262 hours). Separation buffer: $50 \mathrm{mM}$ MES (pH 6.5), $2 \mathrm{M}$ urea, and $20 \mathrm{mM} \mathrm{CM}-\gamma-\mathrm{CD}$ with $10 \mathrm{mM} \beta-C D$. Injection by pressure, 50 mbar for $1 \mathrm{~s}$. Capillary, $58.5 \mathrm{~cm}(50 \mathrm{~cm}$ to the detector $) \times 50 \mu \mathrm{m}$ i. d. Temperature, $45^{\circ} \mathrm{C}$. Applied voltage, $20 \mathrm{kV}(\sim 97 \mu \mathrm{A})$; UV detection at $230 \mathrm{~nm}$.

As an example, Figure 1 shows the electropherograms obtained during the biodegradation of a mixture of PCBs 149 and 95 at three different incubation times (0, 94, and 262 hours). Using as reference the results obtained from control samples analyses ( $\%$ of degradation $=0)$ the degree of degradation of PCBs was calculated for two individual experiments injected by duplicate for the averaged incubation times of 22, 46, 71, 93, 165, 213, and 262 hours. Table 2 shows the degree of degradation calculated for the eight chiral PCBs studied at the above-mentioned incubation times. The biodegradation process has

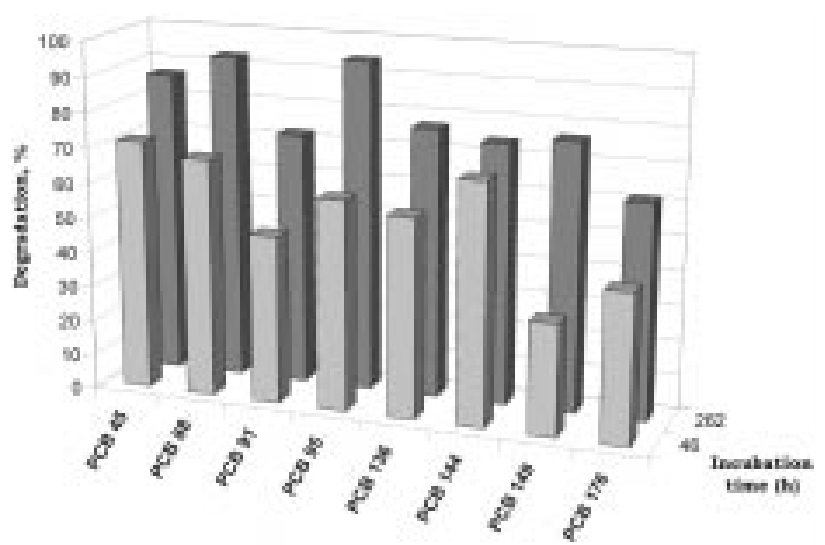

Figure 2. Comparison of the degree of biodegradation obtained at 46 and 262 hours for the eight chiral PCBs studied.

been followed until a degradation degree higher than $61 \%$ for all chiral PCBs was reached (from 61 to $94 \%$ ). It could be observed that the degradation rate is different depending on the studied PCB. As an example, at an incubation time of 48 hours the difference of the degree of biodegradation between the most degraded congeners and the least degraded congeners is close to $40 \%$ while at 262 hours this difference is about $33 \%$. In general, it can be observed that the degradation is favoured for the least chlorinated PCBs. A comparison of the degree of degradation obtained at 46 and 262 hours for all PCBs studied is shown in Figure 2. It can be seen that at low incubation time $(46 \mathrm{~h})$ the highest degree of degradation corresponds to the least chlorinated PCBs (four and five chlorine atoms) whereas the lowest degrees of degradation are obtained for PCBs 149 and 176 with six and seven chlorine atoms, respectively.

\subsection{Study of the stereoselectivity of the biodegradation process}

The investigation of the stereoselectivity of the biodegradation process has been carried out measuring the atropisomeric ratio for seven of the eight chiral PCBs studied due to the low chiral resolution obtained for the PCB 176

Table 2. Degradation, in \%, of the PCBs analysed at different incubation times.

\begin{tabular}{ccccccccc}
\hline $\begin{array}{c}\text { Incubation } \\
\text { time }(\mathrm{h})\end{array}$ & $\begin{array}{c}\text { PCB 45 } \\
\text { (RSD,\%) }\end{array}$ & $\begin{array}{c}\text { PCB 88 } \\
\text { (RSD,\%) }\end{array}$ & $\begin{array}{c}\text { PCB 91 } \\
\text { (RSD,\%) }\end{array}$ & $\begin{array}{c}\text { PCB 95 } \\
\text { (RSD,\%) }\end{array}$ & $\begin{array}{c}\text { PCB 136 } \\
\text { (RSD,\%) }\end{array}$ & $\begin{array}{c}\text { PCB 144 } \\
\text { (RSD,\%) }\end{array}$ & $\begin{array}{c}\text { PCB 149 } \\
\text { (RSD,\%) }\end{array}$ & $\begin{array}{c}\text { PCB 176 } \\
(\text { RSD,\%) }\end{array}$ \\
\hline 22 & $38.2(0.0)$ & $62.3(0.0)$ & $5.5(0.0)$ & - & $63.7(0.0)$ & $71.7(0.0)$ & - & $52.9(9.6)$ \\
46 & $71.5(1.7)$ & $68.0(2.7)$ & $47.9(1.5)$ & $60.1(0.0)$ & $57.0(6.3)$ & $69.0(3.5)$ & $31.7(2.8)$ & $42.4(7.9)$ \\
71 & $82.5(2.2)$ & $85.7(2.2)$ & $57.4(7.1)$ & $61.9(4.8)$ & $76.4(5.6)$ & $74.7(12.2)$ & $30.5(7.3)$ & $71.7(0.0)$ \\
93 & $88.7(0.0)$ & $84.4(8.3)$ & $52.1(3.1)$ & $84.8(7.9)$ & $73.5(9.2)$ & - & $61.2(15.0)$ & - \\
165 & - & $87.8(3.6)$ & $63.6(5.7)$ & $87.1(10.3)$ & $71.9(11.8)$ & - & $67.0(13.9)$ & - \\
213 & $80.9(0.1)$ & $92.3(1.6)$ & $60.2(13.5)$ & $94.1(1.6)$ & $83.1(4.2)$ & $78.5(0.0)$ & $77.1(9.9)$ & - \\
262 & $86.8(2.6)$ & $93.1(0.2)$ & $72.2(0.8)$ & $94.4(1.1)$ & $77.0(3.4)$ & $74.3(0.0)$ & $76.5(2.7)$ & $61.3(0.0)$ \\
\hline
\end{tabular}


Table 3. Atropisomeric ratio obtained for the seven PCBs which atropisomers were baseline separated along the incubation period (Atropisomeric ratio = corrected area of the first-migrating atropisomer/corrected area of the second-migrating atropisomer).

\begin{tabular}{|c|c|c|c|c|c|c|c|}
\hline $\begin{array}{l}\text { Incubation } \\
\text { time (h) }\end{array}$ & $\begin{array}{l}\text { PCB } 45 \\
(\mathrm{RSD}, \%)\end{array}$ & $\begin{array}{l}\text { PCB } 88 \\
(\mathrm{RSD}, \%)\end{array}$ & $\begin{array}{l}\text { PCB } 91 \\
\text { (RSD,\%) }\end{array}$ & $\begin{array}{l}\text { PCB 95 } \\
\text { (RSD,\%) }\end{array}$ & $\begin{array}{l}\text { PCB } 136 \\
(\mathrm{RSD}, \%)\end{array}$ & $\begin{array}{l}\text { PCB } 144 \\
(\mathrm{RSD}, \%)\end{array}$ & $\begin{array}{l}\text { PCB } 149 \\
\text { (RSD,\%) }\end{array}$ \\
\hline 0 & 1.05 (1.8) & $1.01(0.8)$ & $1.02(0.0)$ & $1.01(5.9)$ & 1.05 (1.8) & 1.05 (1.8) & $1.04(1.6)$ \\
\hline 22 & - & $1.04(0.0)$ & $1.07(0.0)$ & - & $1.00(0.0)$ & $1.10(2.4)$ & $1.04(0.0)$ \\
\hline 46 & 1.07 (7.9) & $1.02(1.4)$ & $1.06(0.0)$ & $1.09(0.0)$ & $1.03(4.7)$ & $1.08(2.7)$ & 1.03 (3.4) \\
\hline 71 & $1.04(3.1)$ & $1.03(2.9)$ & $1.03(3.1)$ & 1.09 (2.8) & $0.99(5.1)$ & $1.04(0.2)$ & $1.11(0.0)$ \\
\hline 93 & $1.05(0.0)$ & 1.05 (5.6) & 1.05 (2.5) & $1.05(0.0)$ & $1.03(0.2)$ & - & 1.09 (3.0) \\
\hline 165 & - & $1.02(6.4)$ & $1.05(0.9)$ & $1.11(0.0)$ & $1.05(0.2)$ & $1.01(5.6)$ & $1.11(4.2)$ \\
\hline 213 & $0.99(6.0)$ & 1.02 (7.3) & 1.05 (2.8) & $1.09(0.0)$ & 0.97 (1.6) & $1.06(0.7)$ & $1.13(5.1)$ \\
\hline 262 & $1.02(2.0)$ & $1.02(0.0)$ & $1.03(4.1)$ & $1.11(0.3)$ & $1.00(0.9)$ & $1.04(0.0)$ & $1.13(2.3)$ \\
\hline
\end{tabular}

(see Table 1). The atropisomeric ratio has been calculated comparing the peak areas of the first-migrating atropisomer and the second one in each case. Table 3 displays the results obtained for the atropisomeric ratio for the seven chiral PCBs along the incubation period. In general, it can be observed a slight deviation of the atropisomeric ratio values from the expected value for a racemic mixture (atropisomeric ratio $=1$ ) which could be attributed to experimental errors. Although for PCB 149 a very slight increase in the atropisomeric ratio was observed with increasing incubation times (atropisomeric ratio increased from 1.04 to 1.13) these variations could be considered as lying within the experimental error. In conclusion, results have revealed no significant stereoselective degradation by the soil bacterium under the experimental conditions employed in this work.

\section{Conclusions}

In this work we have studied the application of electrokinetic chromatography (EKC) using a dual cyclodextrin system to monitor the biodegradation process of eight chiral PCBs $(45,88,91,95,136,144,149$, and 176) during more than 250 hours and to study the stereoselectivity of this process for each congener.

A high degree of degradation (from $61 \%$ to $94 \%$ ) by the naturally occurring soil bacterium used in this work has been achieved for all the PCBs studied, the degradation rate being higher for the least chlorinated congeners (PCBs 45 and PCB 88).

No stereoselective biodegradation has been observed for the seven chiral PCBs whose atropisomers have been baseline separated (PCBs 45, 88, 91, 95, 136, 144, 149).

\section{Acknowledgments}

The authors thank the Comunidad Autónoma de Madrid (Spain) for projects $07 \mathrm{M} / 0640 / 1997$ and $07 \mathrm{M} / 0049$ / 1998.

\section{References}

[1] M.J. González, M.A. Fernández, L.M. Hernández, Arch. Environ. Contam. Toxicol. 1991, 20, 343-348.

[2] G. Font, J. Mañes, J.C. Moltó, Y. Picó, J. Chromatogr. A 1996, 733, 449-471.

[3] M.T. Harju, P. Haglund, Fresenius Z. Anal. Chem. 1999, 364, 219-223.

[4] J. Faller, H. Hühnerfuss, W.A. König, R. Krebber, P. Ludwig, Environ. Sci. Technol. 1991, 25, 676-678.

[5] H.R. Buser, M.D. Müller, C. Rappe, Environ. Sci. Technol. 1992, 26, 1533-1540.

[6] J.S. Waid, in: PCBs and the Environment, Volume II, CRC Press, Inc., Florida 1986.

[7] A. Glausch, J. Hahn, V. Schurig, Chemosphere 1995, 30, 2079-2085

[8] A. Glausch, G.P. Blanch, V. Schurig, J. Chromatogr. A 1996, 723, 399-404.

[9] G.P. Blanch, A. Glausch, V. Schurig, R. Serrano, M. J. González, J. High Resol. Chromatogr. 1996, 19, 392396.

[10] R. Looser, K. Ballschmiter, J. Chromatogr. A 1999, 836, 271-284.

[11] L. Ramos, L.M. Hernández, M.J. González, Anal. Chem. 1999, 71, 70-77.

[12] J.L.M. Vidal, M.C.P. Espada, A.G. Frenich, F.J. Arrebola, J. Chromatogr. A 2000, 867, 235-245.

[13] C.S. Wong, A.W. Garrison, J. Chromatogr. A 2000, 866, 213-220.

[14] B. Chankvetadze, in: Capillary Electrophoresis in Chiral Analysis, John Wiley \& Sons, Chichester, 1997.

[15] M.L. Marina, I. Benito, J.C. Díez-Masa, M.J. González, Chromatographia 1996, 42, 269-272.

[16] M.L. Marina, I. Benito, J.C. Díez-Masa, M.J. González, J. Chromatogr. A 1996, 752, 265-270.

[17] A.L. Crego, M.A. García, M.L. Marina, J. Microcol. Sep. 2000, 12, 33-40. 
[18] A.L. Crego, M.J. González, M.L. Marina, Electrophoresis 1998, 19, 2113-2118.

[19] C. García-Ruiz, Y. Martín Biosca, A.L. Crego, M.L. Marina, J. Chromatogr. A 2001, 910, 157-164.

[20] J. Ballschmitter, M. Zell, Fresenius Z. Anal. Chem. 1980, 302, 20-31.
[21] I. Sierra, J.L. Valera, M.L. Marina, F. Laborda, I $V^{\text {th }}$ International Symposium on the Interface between Analytical Chemistry and Microbiology-ISIAM at Trégastel (France) June 4-7, 2000.

[22] J.P. Schaeper, M.J. Sepaniak, Electrophoresis 2000, $21,1421-1429$ 